\title{
A 60-year-old female with asymptomatic total thoracic-abdominal aortic aneurysm
}

\author{
Jian-Ying Deng ${ }^{1}$ and Wei Liu ${ }^{1}$ \\ ${ }^{1}$ Affiliation not available
}

May 3, 2021

\begin{abstract}
Total thoracic-abdominal aortic aneurysm is a rare disease in cardiovascular surgery, with high surgical risk and high mortality. Surgery is considered the most effective treatment for total aortic aneurysms. Recently, our group admitted a 60-year-old female patient with asymptomatic full thoracic-abdominal aortic aneurysm, and successfully performed staged surgery, namely Bentall + Sun's operation in the first stage and thoraco-abdominal aortic replacement in the second stage. The results of the operation were satisfactory.
\end{abstract}

A 60-year-old female with asymptomatic total thoracic-abdominal aortic aneurysm aneurysm of the total aorta Jianying Deng ${ }^{1}, \mathrm{MD}$ Wei $\mathrm{Liu}^{2}, \mathrm{MD}$

${ }^{1}$ Department of Cardiovascular Surgery,Chongqing Kanghua Zhonglian Cardiovascular Hospital, Chongqing 400015, China

${ }^{2}$ Department of Cardiac Surgery, DeltaHealth Hostital, Shanghai 201720, China

Correspondence: Jianying Deng, Department Of Cardiovascular Surgery,Chongqing Kanghua Zhonglian Cardiovascular Hospital, No. 168, Haier Road, District of Jiang Bei, Chongqing 400015, China. e-mail: 65673171@qq.com

Abstract Total thoracic-abdominal aortic aneurysm is a rare disease in cardiovascular surgery, with high surgical risk and high mortality. Surgery is considered the most effective treatment for total aortic aneurysms. Recently, our group admitted a 60-year-old female patient with asymptomatic full thoracic-abdominal aortic aneurysm, and successfully performed staged surgery, namely Bentall + Sun's operation in the first stage and thoraco-abdominal aortic replacement in the second stage. The results of the operation were satisfactory.

Key words aortic aneurysm; staged repair; surgery

\section{Introduction}

Thoracic-abdominal aortic aneurysm refers to aortic aneurysm lesions crossing the diaphragm, involving the thoracic aorta and the abdominal aorta. Therefore, the thoracic and abdominal aorta should be repaired. In recent years, despite the vigorous development of endovascular treatment, surgical repair technique is still an important method for successful treatment of thoracic-abdominal aortic aneurysms.

\section{Case report}

A 60-year-old female was admitted to our hospital with asymptomatic complex aneurysm of the entire thoracic-abdominal aorta. Two month ago, the patient's routine chest x-ray revealed a huge mass in the 
upper mediastinum (Figure 1A), and further chest CT revealed an aneurysm of the total thoracic-abdominal aorta (Figure 1B). For further surgical treatment, she was transferred to our hospital.

On admission, his vital signs were stable, no fever, no chest and abdominal pain. Blood pressure, 132/78 $\mathrm{mmHg}$; heart rate, 80 beats/min; temperature, 36.4; respiratory rate, 16 beats/min; arterial oxygen saturation on room air, $97 \%$. The height is $162 \mathrm{~cm}$, the weight is $65 \mathrm{~kg}$, and the body mass index is $24.8 \mathrm{~kg} / \mathrm{m}^{2}$. The patient's eye vision is normal. There was no abnormal growth in the bones of her limbs. She has a history of hypertension with well controlled. She denies that there are genetic diseases such as Marfan syndrome in her family, and she denies her personal history of drug abuse and promiscuity.

Laboratory test results are as follows: total cholesterol is $5.8 \mathrm{mmol} / \mathrm{L}$, triglyceride is $2.09 \mathrm{mmol} / \mathrm{L}$, and uric acid is $426 \mathrm{umol} / \mathrm{L}$.Transthoracic echocardiography revealed that the patient's aortic sinus was widened (maximum diameter, $50 \mathrm{~mm}$ ) and the aortic valve was moderate regurgitation. Aortic CTA suggests aortic aneurysm, lesions involving the ascending aorta, aortic arch, thoracic-abdominal aorta, mural thrombus in the aortic arch and abdominal aorta, multiple penetrating ulcers in the thoracic-abdominal aorta, $58 \mathrm{~mm}$ at the widest part of the ascending aorta, aortic arch $59 \mathrm{~mm}$ in diameter, $72 \mathrm{~mm}$ in the widest part of the thoracic aorta, and $57 \mathrm{~mm}$ in the widest part of the subrenal abdominal aorta (Figure 1B).

Given the patient's total thoracic-abdominal aortic aneurysm is very complicated, after thorough discussion and fully communication with the patient and family members, our group believes that the risk of onestage total thoracic-abdominal aortic replacement surgery is very high. We finally decided to implement two-staged surgery, namely Bentall procedure and Sun's operation in the first-stage and thoraco-abdominal aortic replacement in the second-stage. After the first-stage Bentall procedure and Sun's operation was successfully performed, a re-examination of CTA showed that the lesions of aneurysm in the aortic root, ascending aorta, and aortic arch were well treated (Figure 2). One month after discharge, in order to avoid the rupture of the residual thoracic-abdominal aortic aneurysm, we performed the second-stage surgery namely total thoracic-abdominal aortic replacemen for the patient. Fortunately, the patient tolerated the operation well, and the postoperative CT scan showed that the total thoracic-abdominal aortic aneurysm was completely removed ( Figure 3 ).

\section{Discussion}

Patients with thoracic-abdominal aortic aneurysm usually have no symptoms until the lesion is severe enough to cause compression of the surrounding organs or dissection or rupture ${ }^{1}$. Therefore, the diagnosis of thoracicabdominal aortic aneurysm is often accidentally discovered during imaging examinations due to other unrelated medical diseases. This patient has no symptoms, and a huge mass in the upper mediastinum was discovered during routine chest radiographs. In addition, once the patient has symptoms, it often indicates that the aneurysm is about to rupture. The most common symptoms are pain in the chest, abdomen, and back. This pain is due to the compression of adjacent organs by an aortic aneurysm, or it may be due to dissection or rupture. Clinically, these symptoms should be highly vigilant.

The diagnosis of thoracic-abdominal aortic aneurysm mainly relies on X-ray imaging ${ }^{2}$. At present, CTA and 3 -dimensional reconstruction of the aorta have become the gold standard for preoperative imaging. CTA plays a vital role in formulating surgical strategies, especially when endovascular treatment is planned. Performing 3-dimensional reconstruction while observing the axial section will help to understand the anatomy of the aneurysm in detail. This patient's CTA was intuitive and provided detailed information for disease diagnosis and surgical design.

The entire thoracic-abdominal aneurysm involves start at the root of the aorta to the end at the bifurcation of the aorta-iliac artery. Surgery methods include staged total aortic replacement, elephant trunk staged total aortic replacement, or one-stage total aortic replacement ${ }^{3}$. One-stage total aortic replacement is the most complicated operation in aortic surgery, including the replacement and repair of the aortic root, ascending part, arch, descending thoracic aorta and abdominal aorta, involving the protection of all important organs of the human body, such as heart, brain, spinal cord, lungs, kidneys and liver. In addition, the operation time is long, the trauma is huge, and the postoperative complications and mortality are very high ${ }^{4}$. The 
patient is an older female and has obvious aortic calcification. In order to reduce the risk of surgery, we finally chose two-staged total aortic replacement with a stent elephant trunk, and the clinical results were satisfactory.

In conclusion, patients with total thoracic-abdominal aortic aneurysm may not have typical clinical symptoms and require a careful and comprehensive physical examination and related auxiliary examinations by clinicians. Staged repair of total thoracic-abdominal aortic aneurysms is still a safe and effective treatment.

Conflict of interest: All authors declare that there is no conflict of interest.

Ethical review: This study has been approved by the ethics committee of the Chongqing Kanghua Zhonglian Cardiovascular Hospital and DeltaHealth Hostital, and has been recognized as exempt from ethical review.

Informed consent statemen t: Informed consent and ethical approval were waived for this report, which contains no patient identifiable data.

References

1. Massimo CG, Presenti LF, Favi PP, Crisci C, Guadrónet EAC. Simultaneous total aortic replacement from valve to bifurcation:experience with 21 cases. Ann Thorac Surg. 1993; 56(5):1110-1116.

2. LeMaire SA, Carter SA, Coselli JS. The elephant trunk technique for staged repair of complex aneurysms of the entire thoracic aorta. Ann Thorac Surg. 2006; 81(5):1561-1569.

3. Hu XP, Chang Q, Zhu JM, Yu CT, Liu ZG, Sun LZ. One-stage total or subtotal aortic replacement. Ann Thorac Surg. 2006; 82(2):542-546.

4. Safi HJ, Miller 3rd CC, Estrera AL, et al. Staged repair of extensive aortic aneurysms: morbidity and mortality in the elephant trunk technique. Circulation. 2001; 104(24):2938-2942.

Figure Legends:

Figure 1. Chest radiograph showed a huge mass in the upper mediastinum (A); CTA showed a total aortic aneurysm, and the lesion involved the aortic root, ascending aorta, aortic arch, and the entire thoracoabdominal aorta. The aneurysm is tortuous and deformed, mural thrombus can be seen in the aortic arch and abdominal aorta, multiple penetrating ulcers can be seen also in the thoracic and abdominal aorta, and the widest part of the aneurysm is $72 \mathrm{~mm}$ in diameter (B).

Figure 2. A re-examination CT of the patient after the first-stage operation showed that the lesions of aortic root, ascending aorta, aortic arch, and descending aorta were eliminated and the rest aneurysm of thoracic-abdominal aorta can be seen clearly.

Figure 3. The patient successfully underwent the second-stage surgery (thoracic-abdominal aortic replacement) and a re-examination CT showed that the artificial vessel was smooth in shape, the entire thoracicabdominal aortic aneurysm was completely removed, and the revascularization of important organs was satisfactory. 


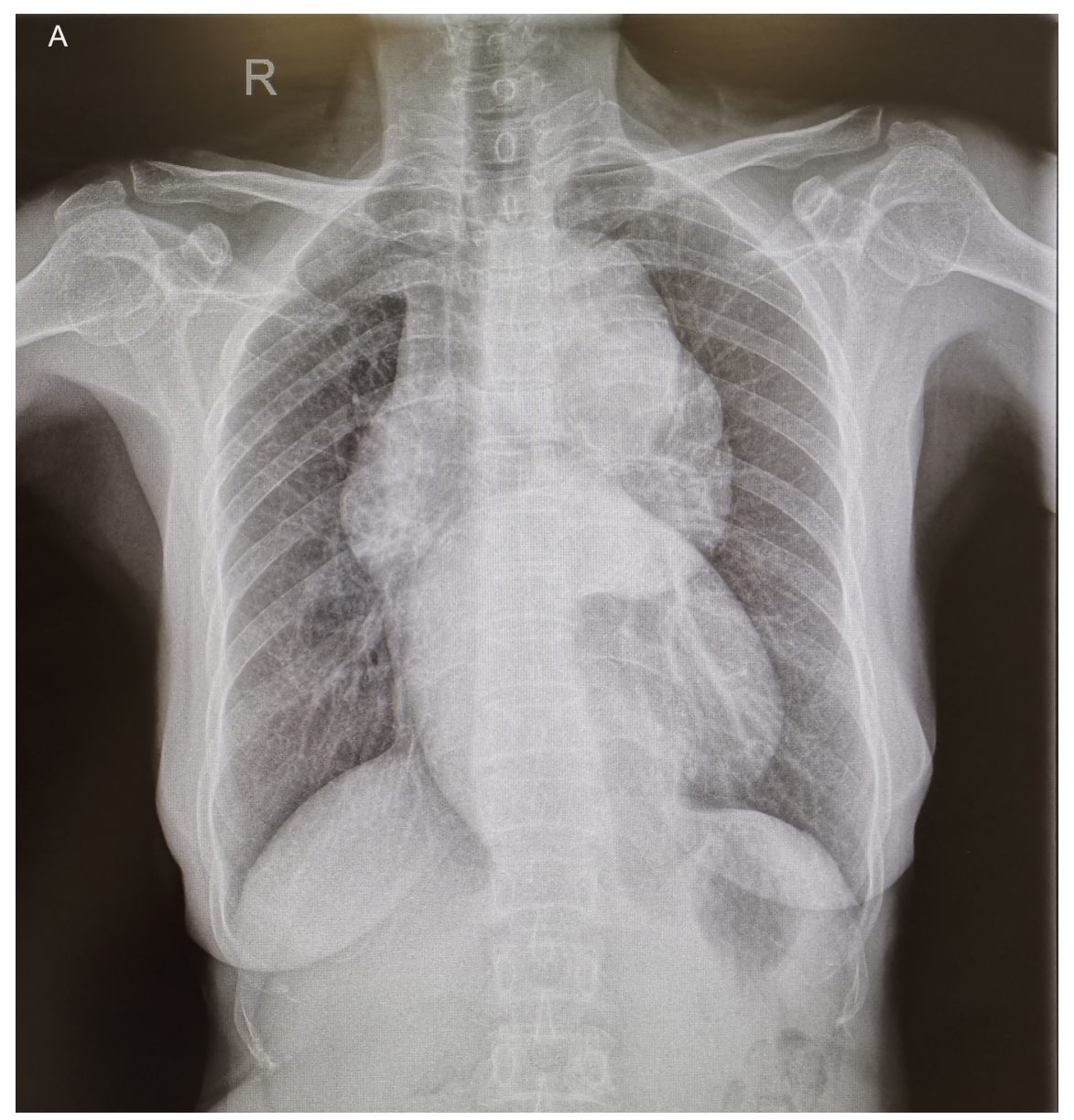




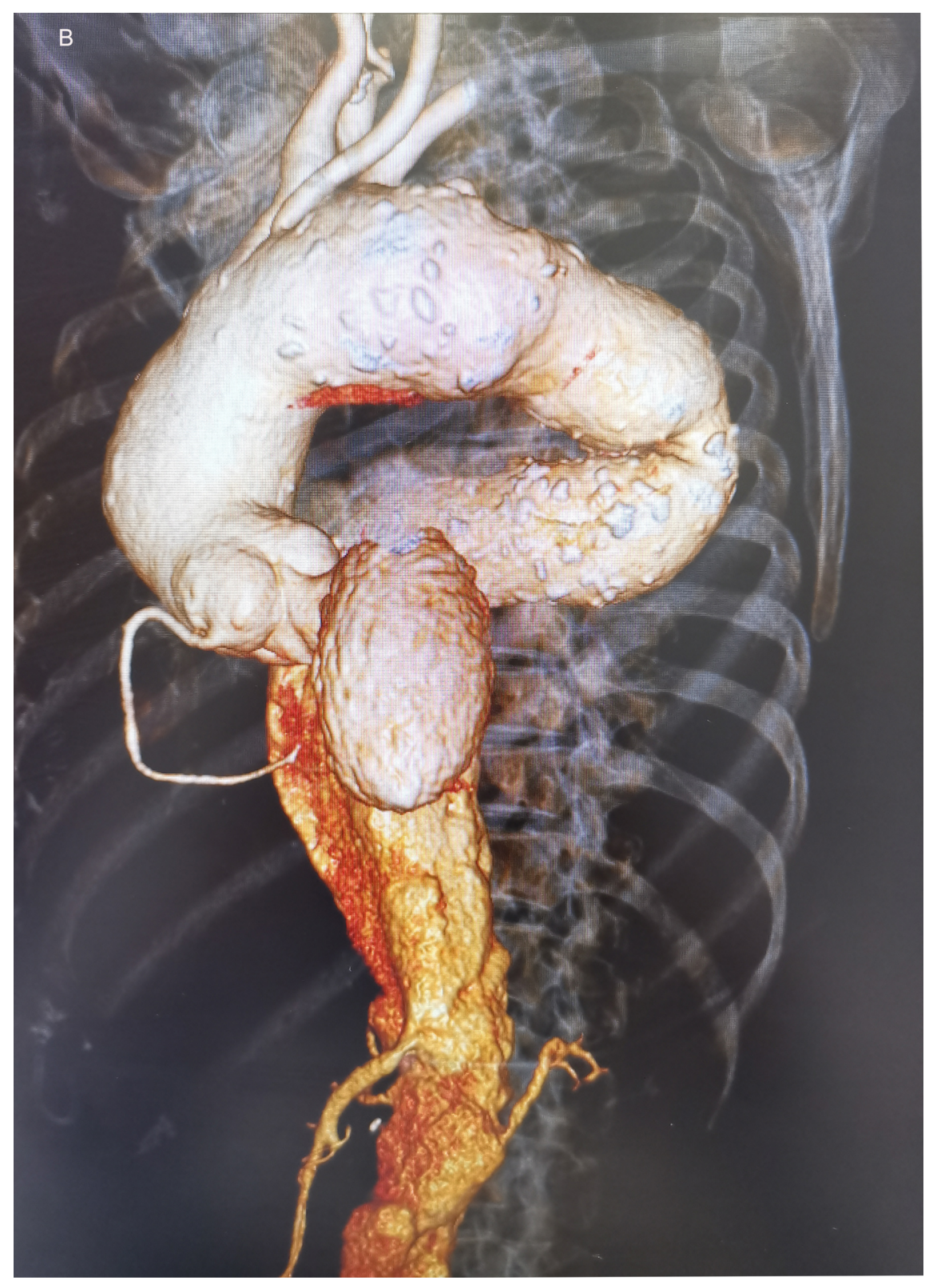




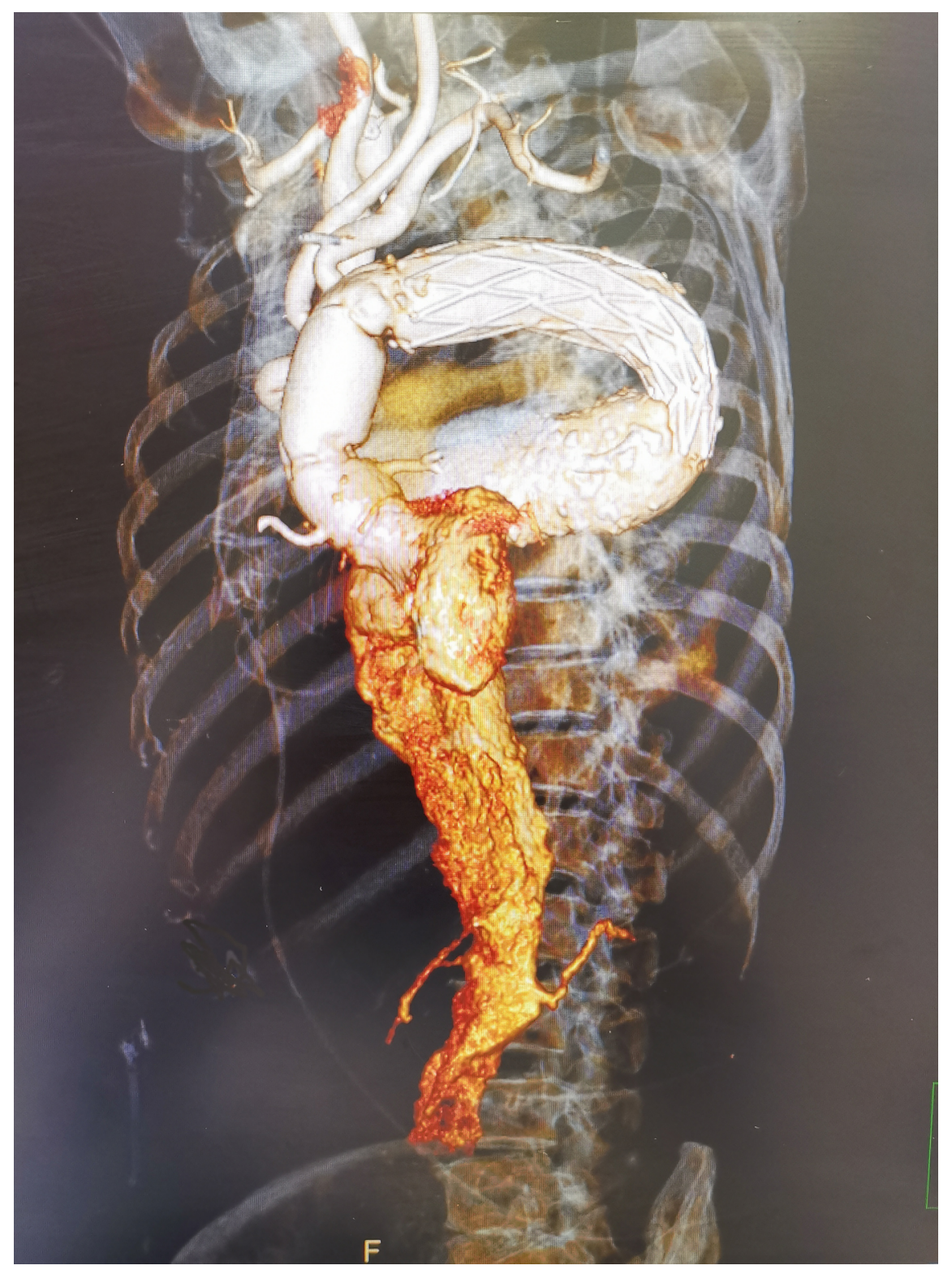




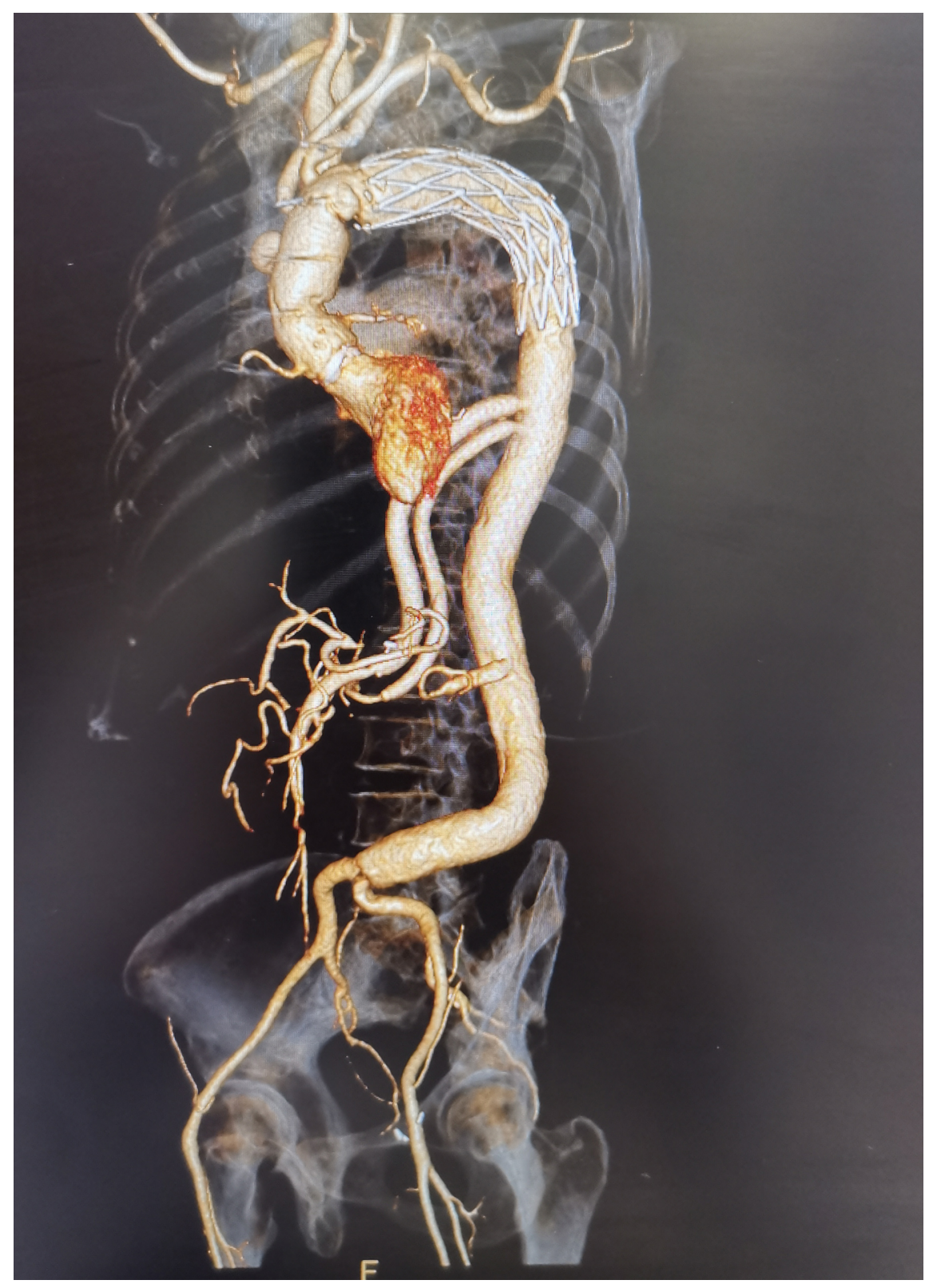

